AIAA-99-2144

\title{
PROPULSION THROUGH ELECTROMAGNETIC SELF-SUSTAINED ACCELERATION
}

\author{
Vesselin Petkov \\ Physics Department, Concordia University \\ 1455 De Maisonneuve Boulevard West \\ Montreal, Quebec, Canada H3G 1M8 \\ E-mail: vpetkov@alcor.concordia.ca
}

\begin{abstract}
As is known the repulsion of the volume elements of an uniformly accelerating charge or a charge supported in an uniform gravitational field accounts for the electromagnetic contribution to the charge's inertial and gravitational mass, respectively. This means that the mutual repulsion of the volume elements of the charge produces the resistance to its accelerated motion. Conversely, the effect of electromagnetic attraction of opposite charges enhances the accelerated motion of the charges provided that they have been initially uniformly accelerated or supported in an uniform gravitational field. The significance of this effect is that it constitutes a possibility of altering inertia and gravitation.
\end{abstract}

\section{Introduction}

In 1881 J. J. Thomson [1] first realized that a charged particle was more resistant to being accelerated than an otherwise identical neutral particle. His observation marked the origin of the concept of electromagnetic mass of charged particles. This concept was developed in a full theory mostly by Heaviside [2], Searle [3], Lorentz 4, 5], Poincaré [6, 8, Abraham [8], Fermi [9, 10] and Rohrlich [11, 12]. It follows from this theory that it is the unbalanced repulsion of the volume elements of an accelerating charged particle that causes the resistance to its acceleration known as inertia. Alternatively, the unbalanced attraction of accelerating opposite charges results in further enhancement of their acceleration. By the equivalence principle the opposite effects of resistance to like charges' acceleration and increase of unlike charges' acceleration

\footnotetext{
${ }^{1}$ The mutual repulsion of two inertial like charges is completely balanced and therefore there is no net force acting on the charges.
}

resulting from the unbalanced repulsion of like charges and the unbalanced attraction of unlike charges, respectively should also occur when the charges are in a gravitational field. The equivalence principle requires that these effects be present in a gravitational field as well but does not provide any insight into what causes them there. The answer to this question is that it is a spacetime anisotropy around massive bodies that causes those effects. It manifests itself in the anisotropy of the velocity of electromagnetic signals (for short - the velocity of light). Since it is now believed that the anisotropy of the velocity of light around massive bodies results from the curvature of spacetime, here we shall discuss two independent results which indicate that the correct interpretation of general relativity is in terms of spacetime anisotropy, not spacetime curvature. While the first result just shows that there is no need for spacetime curvature since spacetime anisotropy alone accounts for all inertial and gravitational effects, the second one directly demonstrates that the standard curved-spacetime interpretation of general relativity contradicts the gravitational redshift experiments. The implication that there is no spacetime curvature is crucial not only for understanding and possible utilization of the effects discussed but also for gaining deeper insight into the nature of inertia and gravitation. One far-reaching consequence from the anisotropy of spacetime is that inertia and gravitation can (at least in principle) be electromagnetically manipulated.

It is the anisotropy of spacetime that causes the phenomena traditionally called inertia and gravitation. An analysis of the classical electromagnetic mass theory in conjunction with general relativity leads to the conclusion that (i) gravitational attraction is caused by the anisotropy of spacetime around massive objects and (ii) inertia (and inertial mass) as described in an accelerating reference frame originates from the space- 
time anisotropy in that frame [13, 14]. The essence of this analysis is as follows. Consider a classical2 electron in the Earth's gravitational field. Due to the anisotropy of the velocity of light in a non-inertial reference frame (supported in the Earth's gravitational field) the electric field of an electron on the Earth's surface is distorted which gives rise to a self-force originating from the interaction of the electron charge with

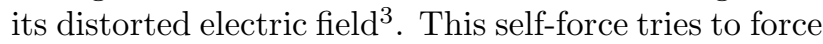
the electron to move downwards and coincides with what is traditionally called the gravitational force. The electric self-force is proportional to the gravitational acceleration $\mathbf{g}$ and the coefficient of proportionality is the mass "attached" to the electron's electric field which proves to be equal to the electron mass. The anisotropy of the speed of light in the Earth's vicinity is compensated if the electron is falling toward the Earth's surface with an acceleration g. In other words, the electron is falling in order to keep its electric field not distorted. A Coulomb (not distorted) field does not give rise to any self-force acting on the electron; that is why the motion of a falling electron is non-resistant (inertial, or geodesic) [. If the electron is prevented from falling it can no longer compensate the anisotropy of the speed of light, its field distorts and as a result a self-force pulling the electron downwards arises. The resistance which an electron offers to being accelerated is similarly described in an accelerating reference frame as caused by the anisotropy of the speed of light there. When the electron accelerates its electric field distorts and the electron resists that deformation. In such a way, given the fact that the speed of light is anisotropic in non-inertial reference frames, all inertial and gravitational effects (including the equivalence of inertial and gravitational mass) of the electron and the other elementary charged particles are fully and consistently accounted for if both the inertial and passive gravitational mass of the elementary charged particles are entirely 6 electromagnetic in origin. The

\footnotetext{
${ }^{2}$ At present quantum mechanical treatment of the electromagnetic mass is not possible since quantum mechanics does not offer a model for the quantum object itself.

${ }^{3}$ That explanation of the origin of the self-force is another way of saying that the force arises from the mutual unbalanced repulsion of the volume elements of the electron charge.

${ }^{4}$ The self-force which starts to act on the electron whenever its electric field distorts effectively resists this distortion. That is why the self-force strives to make the electron move downwards with an acceleration $\mathbf{g}$ in order to compensate the spacetime anisotropy which in turn will eliminate the distortion of the electron's electric field.

${ }^{5}$ It is clear from here that a falling electron does not radiate since its electric field is the Coulomb field and therefore does not contain the radiation $r^{-1}$ terms 13 .

${ }^{6}$ On the one hand, the entirely electromagnetic mass of an elementary charged particle - an electron for example - is sup-
}

gravitational attraction and inertia of all matter can be accounted for as well if it is assumed that there are no elementary neutral particles in nature. A direct consequence from here is that only charged particles or particles that consists of charged constituents possess inertial and passive gravitational mass 7. Stated another way, it is only elementary charges that comprise a body; there is no such fundamental quantity as mass. This means that a body's (inertial or passive gravitational) mass corresponds to the energy stored in the electric fields of all elementary charged particles comprising the body. However, the inertial and passive gravitational mass of a body manifest themselves as such - as a measure of the body's resistance to being accelerated - only if the body is subjected to an acceleration (kinematic or gravitational). This resistance originates from the unbalanced mutual repulsion of the volume elements of every elementary charged constituent of the body. The active gravitational mass of a body proves to be also electromagnetic originating from its charged constituents 8 since there is no mass but only charges. As there is no need for spacetime curvature since all gravitational effects are fully accounted for by the electromagnetic nature of the passive gravitational mass and the anisotropic velocity of light in the vicinity of a massive object 13, 14, it follows that it is the object's charges (and their fields) that cause that anisotropy of spacetime around the object.

One thing concerning the electromagnetic mass the-

ported by the fact that the electromagnetic mass of the classical electron is equal to its observable mass. On the other hand, the electromagnetic mass raises the question of stability of the electron (what keeps its charge together). This question, however, cannot be adequately addressed until a quantum-mechanical model of the electron structure is obtained. An important feature of the electromagnetic mass theory is that the stability problem does not interfere with the derivation of the self-force (acting on a nop-inertial electron) containing the electromagnetic mass [13, 14]. This hints that perhaps there is no real problem with the stability of the electron (as a future quantum mechanical model of the electron itself may find); if there were one it would inevitably emerge in the calculation of the self-force.

${ }^{7}$ It is evident that in this case the electromagnetic mass theory predicts zero neutrino mass and appears to be in conflict with the apparent mass of the $Z^{0}$ boson which is involved in the neutral weak interactions. The resolution of this apparent conflict could lead to either restricting the electromagnetic mass theory (in a sense that not the entire mass is electromagnetic) or reexamining the facts believed to prove (i) that the $Z^{0}$ boson is a fundamentally neutral particle (unlike the neutron), and (ii) that it does possess inertial and gravitational mass if truly neutral.

${ }^{8}$ Given that the inertial and passive gravitational masses are electromagnetic the electromagnetic nature of the active gravitational mass follows immediately since the three kinds of masses are equal. 
ory which is often overlooked should be especially stressed: even if the mass is viewed as only partly electromagnetic, as presently believed, it still follows that inertia and gravitation are electromagnetic in origin but in part. It should be also noted that once the fact of the partly electromagnetic origin of inertia and gravitation is fully realized a thorough analysis of this open issue can be carried out which will most probably lead to the result that electromagnetic interaction is the only cause behind inertia and gravitation which are now regarded as separate phenomena?

The concept of spacetime curvature is in direct contradiction with experiments. A recently obtained result [15] shows that the gravitational redshift contradicts the curved-spacetime interpretation of general relativity. It has not been noticed up to now that both frequency and velocity of a photon change in the gravitational redshift experiment. In such a way the measurement of a change in a photon frequency is in fact an indirect measurement of a change in its local velocity in this experiment. This shows that the local velocity of a photon depends upon its pre-history (whether it has been emitted at the observation point or at a point of different gravitational potential) - a result that contradicts the standard curved-spacetime interpretation of general relativity which requires that the local velocity of light be always $c$ [16]. Therefore the gravitational redshift demonstrates that general relativity cannot be interpreted in terms of spacetime curvature. This situation calls for another interpretation of the mathematical formalism of general relativity. Such a possibility of interpreting the Riemann tensor not in terms of spacetime curvature but in terms of spacetime anisotropy has always existed since the creation of general relativity but received no attention. In such an interpretation the Riemannian geometry describes not a curved but an anisotropic spacetime thus linking gravitation to the anisotropy of spacetime 13].

An additional indication that spacetime around massive bodies is anisotropic (not curved) comes from the following argument. According to the standard

\footnotetext{
${ }^{9}$ Such an analysis will be presented in another paper. The basic idea of this analysis is to demonstrate that it is highly unlikely that Nature has invented two drastically different and independent causes of gravitation - an anisotropic spacetime for elementary charged particles and a curved spacetime for elementary neutral ones (such as the $Z^{0}$ boson if it turns out to be a truly neutral particle). If the mass of the elementary charged particles is regarded as only partly electromagnetic, the phenomenon of gravitation becomes even more complicated. Spacetime must be anisotropic (to account for the gravitational interaction of the electromagnetic part of the mass of the particles) as well as curved (to account for the gravitational interaction of the non-electromagnetic part of the mass of the charged particles).
}

curved-spacetime interpretation of general relativity the gravitational effects observed in a non-inertial reference frame $N^{g}$ on the Earth's surface are caused by the curvature of spacetime originating from the Earth's mass. The principle of equivalence requires that what is happening in $N^{g}$ be happening in a noninertial (accelerating) frame $N^{a}$ as well. The gravitational effects in general relativity include time and length effects in addition to the pre-relativistic ones (falling of bodies and their weight). These, according to the standard interpretation of general relativity, are also caused by the spacetime curvature around the Earth. By the principle of equivalence the time and length effects must be present in $N^{a}$ as well. An inertial observer can explain those effects happening in $N^{a}$ by employing only special relativity [17]. An observer in $N^{a}$, however, can explain them neither by directly making use of the frame's acceleration nor by the anisotropic velocity of light there since the concepts of time and space are more fundamental than the concepts of velocity and acceleration. The noninertial observer in $N^{a}$ has to prove that spacetime in $N^{a}$ is anisotropic due to $N^{a}$ 's acceleration by obtaining the spacetime interval in $N^{a}$. Only then the observer can derive the time and length effects in $N^{a}$ by using the anisotropic spacetime interval there. Therefore the standard interpretation of general relativity leads to a picture involving the principle of equivalence that is not quite satisfying: if one cannot distinguish between the effects in $N^{a}$ and in $N^{g}$ then why is the spacetime in $N^{a}$ anisotropic while in $N^{g}$ it is curved. Taking into account the two results discussed above the picture becomes perfectly consistent: spacetime in both $N^{a}$ and $N^{g}$ is anisotropic (in $N^{a}$ the spacetime anisotropy is caused by the frame's acceleration while in $N^{g}$ it originates from the elementary charges that comprise the Earth).

The result that spacetime is anisotropic (in which the velocity of light is different in different directions) has enormous implications for both understanding the nature of inertia and gravitation and the possibility of controlling them since both inertia and gravitation turn out to be electromagnetic in origin (at least in part $\left(\mathrm{D}^{\mathrm{O}}\right)$. There exist two theoretical possibilities for electromagnetic manipulation of inertia and gravitation:

(i) Changing the anisotropy of spacetime. Since one of the corollaries of the electromagnetic mass theory is that the anisotropy of spacetime around a body is caused by the body's charged constituents (and

\footnotetext{
${ }^{10}$ It is now an established (but unexplainably ignored 18 ) fact from the electromagnetic mass theory that the inertial mass and inertia are at least partly electromagnetic in origin [12, 19].
} 
their electromagnetic fields), the employment of strong electromagnetic fields can create a local spacetime anisotropy which may lead to a body being propelled without being subjected to a direct force.

(ii) Using the spacetime anisotropy. Due to the anisotropic velocity of light in an accelerating reference frame the electromagnetic attraction of the opposite charges of an accelerating electric dipole enhances its accelerated motion - it leads to a self-sustaining accelerated motion perpendicular to the dipole's axis [20]. According to the principle of equivalence an electric dipole supported in an uniform gravitational field should levitate 21]. In such a way a strong electromagnetic attraction between oppositely charged parts of a non-inertial device may lead to its propulsion or at least to a reduction of its mass; thus allowing for the external force that accelerates the device (or the weight of the device) to be reduced.

The possibility of manipulating inertia and gravitation by changing the anisotropy of spacetime was reported in 14. This paper deals with the possibility of altering inertia and gravitation by using the spacetime anisotropy.

\section{Self-Sustained Acceleration}

The equations of classical electrodynamics applied to an accelerating electric dipole show that it can undergo self-sustaining accelerated motion perpendicular to its axis, meaning that not only does the electromagnetic attraction of the opposite charges of a dipole not resist its accelerated motion but further increases it. The application of the principle of equivalence shows that an electric dipole supported in an uniform gravitational field will be also subjected to a self-sustained acceleration which may lead to the dipole's levitation. Here we shall derive this effect in a gravitational field directly without applying the equivalence principle.

Consider a non-inertial reference frame $N^{g}$ supported in a gravitational field of strength $\mathrm{g}$. The gravitational field is directed opposite to the $y$ axis. A dipole with a separation distance $d$ between the two charges is laying along the $x$ axis. Due to the spacetime anisotropy in $N^{g}$ (manifesting itself in the anisotropic velocity of light in $N^{g}$ ) the electric field of the negative charge $-q$ with coordinates $(d, 0)$ at a point with coordinates $(0,0)$, where the positive charge $+q$ is, is distorted [1]

$$
\mathbf{E}_{-+}^{g}=\frac{-q}{4 \pi \epsilon_{o}}\left(\frac{\mathbf{n}_{-+}}{d^{2}}-\frac{\mathbf{g} \cdot \mathbf{n}_{-+}}{c^{2} d} \mathbf{n}_{-+}+\frac{\mathbf{g}}{c^{2} d}\right)
$$

where $\mathbf{n}_{-+}$is a unit vector pointing from the negative charge toward the positive charge and $\mathbf{n}_{-+}=-\hat{\mathbf{x}}$ where $\hat{\mathbf{x}}$ is a unit vector along the $x$ axis. Since $\mathbf{g} \cdot \mathbf{n}_{-+}=0$ ( $\mathbf{g}$ is orthogonal to $\mathbf{n}_{-+}$) the electric field (1) reduces to

$$
\mathbf{E}_{-+}^{g}=\frac{q}{4 \pi \epsilon_{o} d^{2}} \hat{\mathbf{x}}-\frac{q}{4 \pi \epsilon_{o} c^{2} d} \mathbf{g} .
$$

The force with which the negative charge attracts the positive one in the anisotropic spacetime in $N^{g}$ is 13 , 14

$$
\mathbf{F}_{-+}^{g}=q\left(1-\frac{\mathbf{g} \cdot \mathbf{n}_{+-}}{2 c^{2}}\right) \mathbf{E}_{-+}^{g}
$$

where $\mathbf{n}_{+-}$is a unit vector pointing from the positive charge toward the negative charge. Noting that $\mathbf{g} \cdot \mathbf{n}_{+-}=0$ we can write

$$
\mathbf{F}_{-+}^{g}=\frac{q^{2}}{4 \pi \epsilon_{o} d^{2}} \hat{\mathbf{x}}-\frac{q^{2}}{4 \pi \epsilon_{o} c^{2} d} \mathbf{g} .
$$

The first term in (2) is the ordinary force with which the negative charge attracts the positive one. The second term represents the vertical component of the force (2) that is opposite to $\mathbf{g}$ and has a levitating effect on the positive charge.

The calculation of the force with which the negative charge of the dipole is attracted by the positive one gives

$$
\mathbf{F}_{+-}^{g}=-\frac{q^{2}}{4 \pi \epsilon_{o} d^{2}} \hat{\mathbf{x}}-\frac{q^{2}}{4 \pi \epsilon_{o} c^{2} d} \mathbf{g} .
$$

The net (self) force acting on the dipole as a whole is directly obtained from (2) and (3)

$$
\mathbf{F}_{\text {self }}^{g}=\mathbf{F}_{-+}^{g}+\mathbf{F}_{+-}^{g}=-\frac{q^{2}}{2 \pi \epsilon_{o} c^{2} d} \mathbf{g} .
$$

Therefore, unlike the attraction of the charges of an inertial dipole which does not produce a net force acting on the dipole, the mutual attraction of the charges of a dipole in a gravitational field becomes unbalanced

\footnotetext{
${ }^{11}$ This is the electric field of a charge at rest in a gravitational field. If the charge is uniformly accelerated with $\mathbf{a}=-\mathbf{g}$ its electric field at a distance $d$ from the negative charge is 22,23

$$
\mathbf{E}_{-+}^{a}=\frac{-q}{4 \pi \epsilon_{o}}\left(\frac{\mathbf{n}_{-+}}{d^{2}}+\frac{\mathbf{a} \cdot \mathbf{n}_{-+}}{c^{2} d} \mathbf{n}_{-+}-\frac{\mathbf{a}}{c^{2} d}\right) .
$$

This is the electric field as described in an inertial reference frame. The calculation of the electric field in the accelerated frame in which the dipole is at rest gives the same expression due to the anisotropy of spacetime in that frame [13].
} 
and results in a self-force which opposes the dipole's weight. The effect of the self-force (4) on the dipole can be explained in a sense that a fraction of the dipole of mass

$$
m_{a t t}=\frac{q^{2}}{2 \pi \epsilon_{o} c^{2} d}
$$

resulting from the unbalanced attraction of the two charges, is subjected to an acceleration $-\mathbf{g}$ as long as the dipole stays in a gravitational field of strength $\mathbf{g}$. While the mass (5) remains smaller than the dipole mass the effect of the self-force (4) will be a reduction of the dipole mass by $m_{\text {att }}$ since the self-force is opposite to the dipole's weight. When $m_{\text {att }}$ becomes equal to the dipole mass (i.e. when $\mathbf{F}_{\text {self }}^{g}$ becomes equal to the weight of the dipole), the dipole starts to levitate. Further increase of $m_{\text {att }}$ will result in lifting of the dipole.

If the charges of the dipole are an electron and a positron its weight is $\mathbf{F}=2 m_{e} \mathbf{g}$, where $m_{e}$ is the mass of the electron (and the positron). Using the electron electromagnetic mass [5]

$$
m_{e}=\frac{e^{2}}{4 \pi \epsilon_{o} c^{2} r_{0}}
$$

where $r_{0}$ is the classical electron radius, we can calculate the resultant force acting on the dipole supported in a gravitational field

$$
\mathbf{F}_{r e s}=\mathbf{F}+\mathbf{F}_{\text {self }}^{g}=\frac{e^{2}}{2 \pi \epsilon_{o} c^{2}}\left(\frac{1}{r_{0}}-\frac{1}{d}\right) \mathbf{g} .
$$

As seen from (7) the dipole will start to levitate when the separation distance $d$ between its charges becomes equal to $r_{0}$. However, this could hardly be achieved in a laboratory since $r_{0} \sim 10^{-15} \mathrm{~m}$.

Consider now a reference frame $N^{a}$ which is uniformly accelerating with an acceleration $\mathbf{a}=-\mathbf{g}$. Let the dipole be at rest in $N^{a}$ placed in such a way that the acceleration $\mathbf{a}$ is perpendicular to its axis. In a similar fashion to what we have done in the case of a dipole in $N^{g}$ here too can be shown that due to the anisotropic speed of light in $N^{a}$ there is a self-force acting on the dipole as a whole which is given by

$$
\mathbf{F}_{\text {self }}^{a}=\frac{q^{2}}{2 \pi \epsilon_{o} c^{2} d} \mathbf{a}
$$

(the self-force (8) can be directly obtained from (4) by applying the equivalence principle and substituting $\mathbf{g}=-\mathbf{a}$ ). A fraction of the dipole of mass $m_{a t t}$ resulting from the unbalanced attraction of the two charges will be subjected to an acceleration a as long as the whole dipole is experiencing the same acceleration a. This means that the fraction of the dipole of mass $m_{a t t}$ accelerates on its own (due to the unbalanced attraction of the two charges) which results in a reduction of the dipole's resistance to being accelerated by the external force. Therefore in order to maintain the same acceleration a the external force accelerating the dipole should be reduced. Stated another way, the dipole mass is effectively reduced and the resistance which the dipole offers to being accelerated will reduce as well. When $m_{\text {att }}$ becomes equal to the dipole mass the resistance of the dipole to being accelerated will cease and consequently there will be no external force needed to accelerate it. The dipole will continue to maintain its acceleration entirely on its own - it will be in a state of self-sustained accelerated motion. This type of motion resembles the inertial motion of an object: as a free object continues to move with constant velocity until being prevented from doing so, a dipole (initially accelerated by an external force) whose charges and separation distance ensure that $m_{\text {att }}$ is equal to the dipole mass will continue to move with constant acceleration on its own until being prevented from doing so.

If the charges of the dipole are an electron and a positron the external force accelerating the dipole will be

$$
\mathbf{F}_{\text {ext }}=2 m_{e} \mathbf{a} \text {. }
$$

Taking into account (8) the dipole will mantain a constant acceleration if

$$
\mathbf{F}_{\text {ext }}+\mathbf{F}_{\text {self }}^{a}=2 m_{e} \mathbf{a}
$$

Noting that for an electron and a positron the mass in (8) will be

$$
m_{a t t}=\frac{e^{2}}{2 \pi \epsilon_{o} c^{2} d}
$$

we can write

$$
\mathbf{F}_{\text {ext }}=\left(2 m_{e}-m_{a t t}\right) \mathbf{a} .
$$

If we assume that $m_{\text {att }}>2 m_{e}$ (which is unlikely to be achieved since the separation distance $d$ between the charges should be smaller than the dimension $r_{0}$ of the classical electron considered) an external force would be needed to slow down the dipole in order that it maintains its uniform acceleration a.

The effect of mass reduction caused by the mutual attraction of the accelerating dipole's charges can be described in the following way as well. Instead of regarding the self-force (8) as subjecting only a part of the dipole of mass $m_{\text {att }}$ to the acceleration a it is also possible to say that $\mathbf{F}_{\text {self }}^{a}$ subjects the whole dipole of mass $2 m_{e}$ to an acceleration $\mathbf{a}_{a t t}$ originating from the unbalanced attraction between the electron and 
the positron. Then using the electron electromagnetic mass (6) we obtain the relation between $\mathbf{a}_{a t t}$ and $\mathbf{a}$

$$
\frac{e^{2}}{2 \pi \epsilon_{o} c^{2} r_{0}} \mathbf{a}_{a t t}=\frac{e^{2}}{2 \pi \epsilon_{o} c^{2} d} \mathbf{a}
$$

or

$$
\mathbf{a}_{a t t}=\frac{r_{0}}{d} \mathbf{a} .
$$

As seen from (9) the dipole will experience a selfsustained acceleration $\mathbf{a}_{a t t} \geq \mathbf{a}$ if $d \leq r_{0}$.

\section{Conclusion}

At present it does not appear realistic to expect that a self-sustained acceleration of a body (equal or greater than its initial acceleration) can be achieved. However, the possibility for eventual practical applications of the effect of mass reduction can be assessed if macroscopic charge distributions are considered. It appears that most promising will be the use of specially designed capacitors like the commercial ones which consist of alternatively charged layers of metal foil rolled into the shape of a cylinder with the cylinder axis parallel to $\mathbf{a}$ or $\mathbf{g}$. Such capacitors can be charged to large amounts of charge. There are capacitors already available on the market that can carry a charge well above $1 C$. With such (and greater) amounts of charge the experimental testing of the mass reduction effect appears now possible although it is not a real issue since this effect is a direct consequence of the classical electrodynamics when applied to a non-inertial dipole and for this reason there should be no doubt that it will be experimentally confirmed. The purpose of this paper is to demonstrate that the practical applicability of the mass reduction effect may be within reach if proper technological effort is invested.

\section{Acknowledgement}

I would like to thank Dr. G. Hathaway for bringing to my attention the papers of Cornish [20 and Griffiths [21] in a discussion of the effects considered here.

\section{References}

[1] J. J. Thomson, Phil. Mag. 11, 229 (1881).

[2] O. Heaviside, The Electrician 14, 220 (1885).

[3] G. F. C. Searle, Phil. Mag. 44, 329 (1897).
[4] H. A. Lorentz, Proceedings of the Academy of Sciences of Amsterdam 6, 809 (1904).

[5] H. A. Lorentz, Theory of Electrons, 2nd ed. (Dover, New York, 1952).

[6] H. Poincaré, Compt. Rend. 140, 1504 (1905).

[7] H. Poincaré, Rendiconti del Circolo Matematico di Palermo 21, 129 (1906).

[8] M. Abraham, The Classical Theory of Electricity and Magnetism, 2nd ed. (Blackie, London, 1950).

[9] E. Fermi, Phys. Zeits. 23, 340 (1922).

[10] E. Fermi, Atti Acad. Nazl. Lincei 31, 184; 306 (1922).

[11] F. Rohrlich, Am. J. Phys. 28, 639 (1960).

[12] F. Rohrlich, Classical Charged Particles (Addison-Wesley, New York, 1990).

[13] V. Petkov, Ph.D. Thesis, Concordia University, Montreal, 1997.

[14] V. Petkov, 34th AIAA/ASME/SAE/ASEE Joint Propulsion Conference, Cleveland, Ohio, July 12-15, 1998; Paper AIAA-98-3142 (http://xxx.lanl.gov/abs/physics/9805028).

[15] V. Petkov, Found. Phys. Lett., submitted (http://xxx.lanl.gov/abs/gr-qc/9810030).

[16] C. W. Misner, K. S. Thorne and J. A. Wheeler, Gravitation (Freeman, San Francisco, 1973), p. 385 .

[17] L. Schiff, Am. J. Phys. 28, 340 (1960).

[18] P. Pearle, in D. Teplitz, ed. Electromagnetism: Paths to Research (Plenum Press, New York, 1982), p. 213.

[19] J. W. Butler, Am. J. Phys. 37, 1258 (1969).

[20] F. H. J. Cornish, Am. J. Phys. 54, 166 (1986).

[21] D. J. Griffiths, Am. J. Phys. 54, 744 (1986).

[22] J. D. Jackson, Classical Electrodynamics, 3rd ed., (Wiley, New York, 1999), p. 664.

[23] D. J. Griffiths, Introduction to Electrodynamics, 2nd ed., (Prentice Hall, New Jersey, 1989), p. 424. 